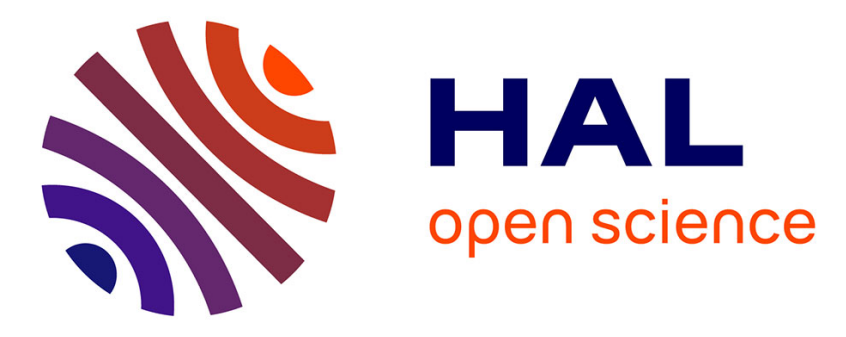

\title{
Selected configuration interaction dressed by perturbation
}

Yann Garniron, Anthony Scemama, Emmanuel Giner, Michel Caffarel, Pierre-François Loos

\section{To cite this version:}

Yann Garniron, Anthony Scemama, Emmanuel Giner, Michel Caffarel, Pierre-François Loos. Selected configuration interaction dressed by perturbation. Journal of Chemical Physics, 2018, 149 (6), pp.064103. 10.1063/1.5044503 . hal-01858534

\section{HAL Id: hal-01858534 https://hal.science/hal-01858534}

Submitted on 28 Jan 2020

HAL is a multi-disciplinary open access archive for the deposit and dissemination of scientific research documents, whether they are published or not. The documents may come from teaching and research institutions in France or abroad, or from public or private research centers.
L'archive ouverte pluridisciplinaire HAL, est destinée au dépôt et à la diffusion de documents scientifiques de niveau recherche, publiés ou non, émanant des établissements d'enseignement et de recherche français ou étrangers, des laboratoires publics ou privés. 


\section{Selected configuration interaction dressed by perturbation}

Cite as: J. Chem. Phys. 149, 064103 (2018); https://doi.org/10.1063/1.5044503

Submitted: 13 June 2018 . Accepted: 26 July 2018 . Published Online: 08 August 2018

Yann Garniron (D), Anthony Scemama (D), Emmanuel Giner (D), Michel Caffarel (D), and Pierre-François Loos

\section{ARTICLES YOU MAY BE INTERESTED IN}

Communication: A mean field platform for excited state quantum chemistry

The Journal of Chemical Physics 149, 081101 (2018); https://doi.org/10.1063/1.5045056

Perspective: Multireference coupled cluster theories of dynamical electron correlation The Journal of Chemical Physics 149, 030901 (2018); https://doi.org/10.1063/1.5039496

Communication: An efficient and accurate perturbative correction to initiator full configuration interaction quantum Monte Carlo

The Journal of Chemical Physics 148, 221101 (2018); https://doi.org/10.1063/1.5037923

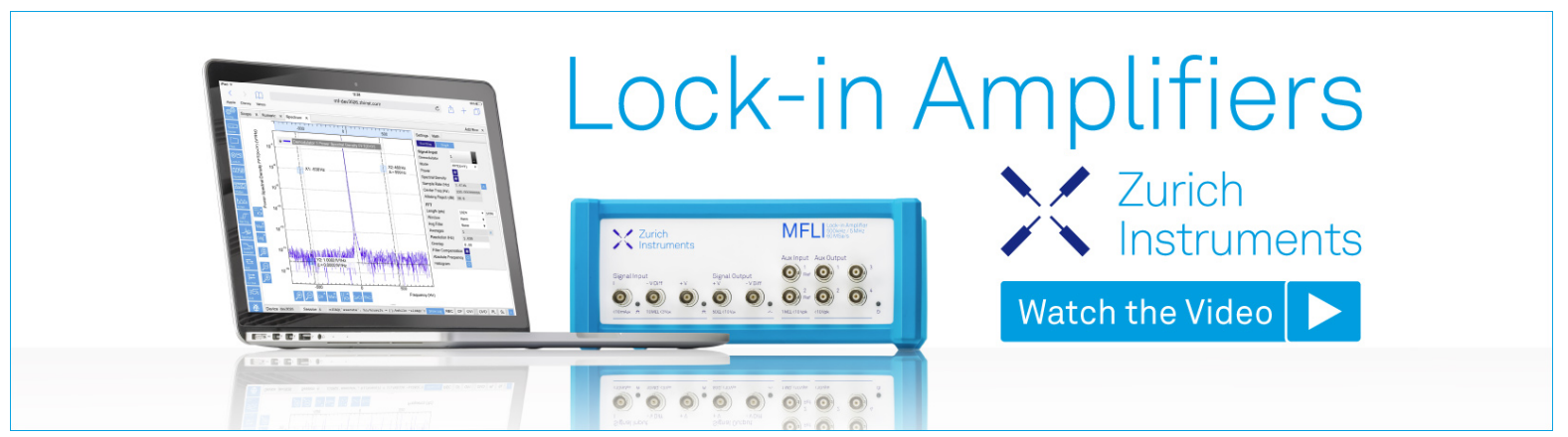




\title{
Selected configuration interaction dressed by perturbation
}

\author{
Yann Garniron, ${ }^{1}$ Anthony Scemama, ${ }^{1}$ Emmanuel Giner, ${ }^{2}$ Michel Caffarel, ${ }^{1}$ \\ and Pierre-François Loos ${ }^{1, a)}$ \\ ${ }^{1}$ Laboratoire de Chimie et Physique Quantiques, Université de Toulouse, CNRS, UPS, Toulouse, France \\ ${ }^{2}$ Laboratoire de Chimie Théorique, Université Pierre et Marie Curie, Sorbonne Université, CNRS, Paris, France
}

(Received 13 June 2018; accepted 26 July 2018; published online 8 August 2018)

\begin{abstract}
Selected configuration interaction (sCI) methods including second-order perturbative corrections provide near full CI (FCI) quality energies with only a small fraction of the determinants of the FCI space. Here, we introduce both a state-specific and a multi-state sCI method based on the configuration interaction using a perturbative selection made iteratively (CIPSI) algorithm. The present method revises the reference (internal) space under the effect of its interaction with the outer space via the construction of an effective Hamiltonian, following the shifted-Bk philosophy of Davidson and co-workers. In particular, the multi-state algorithm removes the storage bottleneck of the effective Hamiltonian via a low-rank factorization of the dressing matrix. Illustrative examples are reported for the state-specific and multi-state versions. Published by AIP Publishing. https://doi.org/10.1063/1.5044503
\end{abstract}

\section{INTRODUCTION}

Recently, selected configuration interaction (sCI) methods have demonstrated their ability to reach, for moderate size basis sets, near full CI (FCI) quality energies for small organic and transition metal-containing molecules. ${ }^{1-13}$ Selecting iteratively the most relevant determinants of the FCI space is an old idea that, to the best of our knowledge, dates back to the pioneering studies of Bender and Davidson ${ }^{14}$ and Whitten and Hackmeyer ${ }^{15}$ in 1969. A few years later, Huron et al. ${ }^{16}$ proposed the so-called CIPSI (Configuration Interaction using a Perturbative Selection made Iteratively) approach to complement the variational sCI energy with a second-order Epstein-Nesbet perturbative correction. This has demonstrated to be a particularly efficient way of approaching the FCI limit. ${ }^{8,11-13,17,18}$ Over these last few years, we have witnessed a resurgence of sCI methods under various variants and acronyms. In short, their main differences lie in the way (i) the determinant selection is done and (ii) the secondorder contribution is computed. The selection can be done purely stochastically as in FCIQMC ${ }^{19}$ or deterministically as in CIPSI or other variants, such as heat-bath $\mathrm{CI},{ }^{7-10}$ adaptive sampling CI (ASCI),${ }^{20-22}$ or iterative CI (ICI). ${ }^{23}$ Similarly, the second-order correction can be computed either purely deterministically or semi-stochastically by a Monte Carlo (MC) sampling. ${ }^{4,8,18}$ Here, we shall use the CIPSI method ${ }^{16}$ to generate the model space, but any other sCI variants could be employed.

For a given electronic state $k$, the ensemble of determinants $|I\rangle$, which constitutes the zeroth-order (normalized) wave function

$$
\left|\Psi_{k}^{(0)}\right\rangle=\sum_{I=1}^{N_{\mathrm{det}}} c_{I k}^{(0)}|I\rangle
$$

\footnotetext{
a)Author to whom correspondence should be addressed: loos@irsamc.upstlse.fr
}

of (variational) zeroth-order energy

$$
E_{k}^{(0)}=\left\langle\Psi_{k}^{(0)}|\hat{H}| \Psi_{k}^{(0)}\right\rangle={ }^{\dagger} \boldsymbol{c}_{k}^{(0)} \boldsymbol{H}^{(0)} \boldsymbol{c}_{k}^{(0)}
$$

(where ${ }^{\dagger} \boldsymbol{c}_{k}^{(0)}$ are the transposed coefficients), defines the (zeroth-order) reference model space or internal space. The remaining determinants of the FCI space belong to the external space or outer space. In particular, the ensemble of determinants $|\alpha\rangle$ connected to $\Psi_{k}^{(0)}$, i.e., $\left\langle\alpha|\hat{H}| \Psi_{k}^{(0)}\right\rangle \neq 0$ and $\left\langle\alpha \mid \Psi_{k}^{(0)}\right\rangle=0$ - the so-called "perturbers"-defines the (first-order) perturbative space, such as

$$
\left|\Psi_{k}^{(1)}\right\rangle=\sum_{\alpha} c_{\alpha k}^{(1)}|\alpha\rangle, \boldsymbol{c}_{k}^{(1)}=\left(E_{k}^{(0)} \mathbf{1}-\boldsymbol{D}^{(1)}\right)^{-1} \boldsymbol{h} \boldsymbol{c}_{k}^{(0)},
$$

where $\mathbf{1}$ is the identity matrix and $\boldsymbol{D}^{(1)}$ is a diagonal matrix with elements $D_{\alpha \alpha}^{(1)}=\langle\alpha|\hat{H}| \alpha\rangle$ and $h_{\alpha I}=\langle\alpha|\hat{H}| I\rangle$. Within CIPSI, the "distance" to the FCI solution is estimated via a second-order Epstein-Nesbet perturbative energy correction

$$
E_{k}^{(2)}=\left\langle\Psi_{k}^{(0)}|\hat{H}| \Psi_{k}^{(1)}\right\rangle={ }^{\dagger} \boldsymbol{c}_{k}^{(0)} \dagger \boldsymbol{h} \boldsymbol{c}_{k}^{(1)} .
$$

The second-order correction (4) has obvious advantages and can be computed efficiently using diagrammatic ${ }^{24}$ or hybrid stochastic-deterministic approaches. ${ }^{8,17,18}$ However, it has also an obvious disadvantage: the internal space is not revised under the effect of its interaction with the outer space. Here, thanks to intermediate effective Hamiltonian theory, ${ }^{25}$ we propose to build and diagonalize an effective Hamiltonian taking into account the effect of the perturbative space. ${ }^{26,27}$ This idea is based on the so-called Bk method, originally proposed by Gershgorn and Shavitt ${ }^{28}$ and later refined and rebranded shifted-Bk (sBk) by Davidson and co-workers. ${ }^{29-38}$ (See also Refs. 39-42.) All these studies lie on the seminal idea of Löwdin on the partition of the FCI Hamiltonian matrix. ${ }^{43}$ Initially, Gershgorn and Shavitt ${ }^{28}$ introduced several approximations, two of them being denoted as Ak and Bk. Both use a partitioning of the CI matrix based on the selection of a dominant subset of (primary) configurations. The Ak method, which is related to earlier work by Claverie, 
Diner, and Malrieu, ${ }^{44}$ estimates the contribution of the configurations left out of the CI expansion, an idea very similar to the computation of the second-order correction [see Eq. (4)]. ${ }^{14,45}$ Compared to the Ak method, the coefficients of the primary configurations are allowed to relax in the Bk method. The different flavours of Bk methods are usually due to the distinct partition of the Hamiltonian matrix and the reference energy used to define the perturbers [see Eq. (3) and discussion below]. ${ }^{26,27,29-42}$

To the best of our knowledge, the shifted-Bk method has never been coupled with CIPSI-like sCI methods. Moreover, in addition to its convergence acceleration to the FCI limit, one of the interesting advantages of shifted-Bk is to provide an explicit revised wave function that one can use, for example, as a trial wave function within quantum Monte Carlo. ${ }^{1,2,5,6,11,13}$ In the present manuscript, we propose both a state-specific and a multi-state formulation which remove the storage bottleneck of the effective Hamiltonian. Furthermore, the present computations are performed semi-stochastically as in our recently proposed hybrid stochastic-deterministic algorithm for the computation of $E^{(2)} \cdot{ }^{17}$ Unless otherwise stated, atomic units are used throughout (see Sec. III).

\section{SHIFTED-Bk}

\section{A. State-specific shifted-Bk}

For a given electronic state $k$, in order to solve the Schrödinger equation $\boldsymbol{H c}_{k}=E_{k} \boldsymbol{c}_{k}$ in the FCI space, the eigenvalue problem may be partitioned as

$$
\left(\begin{array}{ccc}
\boldsymbol{H}^{(0)} & \dagger & \mathbf{0} \\
\boldsymbol{h} & \boldsymbol{H}^{(1)} & { }^{\dagger} \boldsymbol{g} \\
\mathbf{0} & \boldsymbol{g} & \boldsymbol{H}^{(2)}
\end{array}\right)\left(\begin{array}{c}
\boldsymbol{c}_{k}^{(0)} \\
\boldsymbol{c}_{k}^{(1)} \\
\boldsymbol{c}_{k}^{(2)}
\end{array}\right)-E_{k}\left(\begin{array}{c}
\boldsymbol{c}_{k}^{(0)} \\
\boldsymbol{c}_{k}^{(1)} \\
\boldsymbol{c}_{k}^{(2)}
\end{array}\right)=\left(\begin{array}{l}
\mathbf{0} \\
\mathbf{0} \\
\mathbf{0}
\end{array}\right),
$$

where $\boldsymbol{H}^{(2)}$ is the second-order Hamiltonian corresponding to the external configurations excluding the perturbers and $g$ is the coupling matrix between first- and second-order spaces. Equation (5) can be recast as an "effective" Schrödinger equation $\boldsymbol{H}_{k}^{\text {eff }} \boldsymbol{c}_{k}^{(0)}=E_{k} \boldsymbol{c}_{k}^{(0)}$ with the effective Hamiltonian

$$
\boldsymbol{H}_{k}^{\mathrm{eff}}=\boldsymbol{H}^{(0)}+\boldsymbol{\Delta}_{k},
$$

and dressing matrix

$$
\boldsymbol{\Delta}_{k}={ }^{\dagger} \boldsymbol{h}\left[\left(E_{k} \mathbf{1}-\boldsymbol{H}^{(1)}\right)-{ }^{\dagger} \boldsymbol{g}\left(E_{k} \mathbf{1}-\boldsymbol{H}^{(2)}\right)^{-1} \boldsymbol{g}\right]^{-1} \boldsymbol{h} .
$$

Within the state-specific version of the Bk method introduced by Gershgorn and Shavitt, ${ }^{28}$ for each target electronic state $k$, we (i) approximate $\boldsymbol{H}^{(1)}$ by its (diagonal) zeroth-order approximation $\boldsymbol{D}^{(1)}$ and (ii) neglect the influence of the second-order space $\boldsymbol{H}^{(2)}$. Hence, the state-specific Bk dressing matrix is defined as

$$
\boldsymbol{\Delta}_{k}^{\mathrm{Bk}}={ }^{\dagger} \boldsymbol{h}\left(E_{k} \mathbf{1}-\boldsymbol{D}^{(1)}\right)^{-1} \boldsymbol{h},
$$

which naturally yields to a Brillouin-Wigner perturbation approximation. $^{28}$

The shifted-Bk method of Davidson and co-workers ${ }^{29-33}$ still approximates $\boldsymbol{H}^{(1)}$ by its diagonal $\boldsymbol{D}^{(1)}$, but "shifts" (hence the name) the energy at the denominator of Eq. (7) to take into account the influence of the second-order term ${ }^{\dagger} \boldsymbol{g}\left(E_{k} \mathbf{1}-\right.$ $\left.\boldsymbol{H}^{(2)}\right)^{-1} \boldsymbol{g}$; in other words,

$$
E_{k} \mathbf{1}-{ }^{\dagger} \boldsymbol{g}\left(E_{k} \mathbf{1}-\boldsymbol{H}^{(2)}\right)^{-1} \boldsymbol{g} \approx E_{k}^{(0)} \mathbf{1} .
$$

Therefore, the state-specific shifted-Bk dressing matrix is

$$
\boldsymbol{\Delta}_{k}^{\mathrm{sBk}}={ }^{\dagger} \boldsymbol{h}\left(E_{k}^{(0)} \mathbf{1}-\boldsymbol{D}^{(1)}\right)^{-1} \boldsymbol{h},
$$

which leads to the Epstein-Nesbet variant of RayleighSchrödinger perturbation theory. ${ }^{31,32}$ Compared to the $\mathrm{Bk}$ method, its shifted variant has the indisputable advantage of correcting some of the size-consistency error. ${ }^{31}$ However, as expected, the present methodology is only nearly sizeconsistent. Note that the shifted-Bk method is an iterative method as, thanks to the influence of the entire external space, both the zeroth-order coefficients $\boldsymbol{c}_{k}^{(0)}$ and energy $E_{k}^{(0)}$ [given by Eq. (2)] are revised at each iteration.

For small CI expansions, it is possible to store the entire dressed Hamiltonian matrix $\boldsymbol{H}_{k}^{\text {eff }}$ of size $N_{\text {det }} \times N_{\text {det }}$. However, when the $\mathrm{CI}$ expansion gets large, $\boldsymbol{H}_{k}^{\text {eff }}$ becomes too large to be stored in memory. Thankfully, it is not necessary to explicitly build $\boldsymbol{H}_{k}^{\text {eff }}$. Indeed, for large CI expansions, we switch to a Davidson diagonalization procedure ${ }^{46}$ which only requires the computation of the vectors $\boldsymbol{H}^{(0)} \boldsymbol{c}_{k}^{(0)}$ and $\boldsymbol{\Delta}_{k}^{\mathrm{sBk}} \boldsymbol{c}_{k}^{(0)}$ of size $N_{\mathrm{det}}$.

\section{B. Multi-state shifted-Bk}

In a multi-state calculation, one has to adopt a different strategy in order to dress the Hamiltonian for all the target states simultaneously. This is particularly important in practice, for instance, to determine accurate vertical transition energies. An unbalanced treatment of the ground and excited states, even for states with different spatial or spin symmetries, could have significant effects on the accuracy of these energy differences. ${ }^{12}$

For the sake of simplicity, let us assume that our aim is to calculate the dressed energy of the $N_{\text {st }}$ lowest electronic states. For $1 \leq k \leq N_{\text {st }}$, we wish to find a multi-state effective Hamiltonian $\boldsymbol{H}^{\text {eff }}$ and a dressing matrix $\boldsymbol{\Delta}^{\mathrm{sBk}}$, with $\boldsymbol{H}^{\text {eff }}=\boldsymbol{H}^{(0)}$ $+\Delta^{\mathrm{sBk}}$, such that, when applied to the $k$ th state coefficient vector $c_{k}^{(0)}$, one recovers the $k$ th state-specific dressing matrix $\boldsymbol{\Delta}_{k}^{\mathrm{sBk}}$ times the same vector $\boldsymbol{c}_{k}^{(0)}$, i.e.,

$$
\Delta^{\mathrm{sBk}} \boldsymbol{c}_{k}^{(0)}=\boldsymbol{\Delta}_{k}^{\mathrm{sBk}} \boldsymbol{c}_{k}^{(0)}
$$

A solution obeying Eq. (11) is

$$
\boldsymbol{\Delta}^{\mathrm{sBk}}=\sum_{k l} \boldsymbol{\Delta}_{k}^{\mathrm{sBk}} \boldsymbol{c}_{k}^{(0)}\left(\boldsymbol{S}^{-1}\right)_{k l}^{\dagger} \boldsymbol{c}_{l}^{(0)}
$$

where $\left(\boldsymbol{S}^{-1}\right)_{k l}=\left\langle\boldsymbol{c}_{k}^{(0)} \mid \boldsymbol{c}_{l}^{(0)}\right\rangle$. In contrast to the state-specific case, $\boldsymbol{H}^{\text {eff }}$ is non-Hermitian as a consequence of the nonorthogonality of the exact state projections on the model space. ${ }^{25}$ In practice, we have found that a robust algorithm can be defined by symmetrizing the multi-state dressing matrix as

$$
\tilde{\Delta}^{\mathrm{sBk}}=\left({ }^{\dagger} \Delta^{\mathrm{sBk}}+\Delta^{\mathrm{sBk}}\right) / 2 .
$$

The eigenstates being now orthonormal, the dressing matrix reduces to

$$
\Delta^{\mathrm{sBk}}=\delta^{\mathrm{sBk} \dagger} \boldsymbol{c}^{(0)},
$$


which is reminiscent of a low-rank factorization. Here,

$$
\begin{aligned}
\boldsymbol{c}^{(0)} & =\left[\boldsymbol{c}_{1}^{(0)}, \ldots, \boldsymbol{c}_{N_{\mathrm{st}}}^{(0)}\right], \\
\boldsymbol{\delta}^{\mathrm{sBk}} & =\left[\boldsymbol{\Delta}_{1}^{\mathrm{sBk}} \boldsymbol{c}_{1}^{(0)}, \ldots, \boldsymbol{\Delta}_{N_{\mathrm{st}}}^{\mathrm{sBk}} \boldsymbol{c}_{N_{\mathrm{st}}}^{(0)}\right]
\end{aligned}
$$

are both of size $N_{\text {det }} \times N_{\text {st }}$.

Two key remarks are in order here: (i) at first order, the symmetrization error is strictly zero, i.e., ${ }^{\dagger} c_{k}^{(0)}\left(\Delta^{\mathrm{sBk}}-\right.$ $\left.\tilde{\boldsymbol{\Delta}}^{\mathrm{sBk}}\right) \boldsymbol{c}_{k}^{(0)}=0$, and (ii) the symmetrization error becomes vanishingly small for large $\mathrm{CI}$ expansions. Consequently, the symmetrization error can be safely neglected in practice. Our preliminary tests have corroborated these theoretical justifications. Also, it can be further estimated via second-order perturbation theory. However, it requires the energies and coefficients of the entire internal space which is only possible for relatively small CI expansions.

The energies of the first $N_{\mathrm{st}}$ states, $\boldsymbol{E}=\left(E_{1}, \ldots, E_{N_{s t}}\right)$, are obtained by a Davidson diagonalization of the multi-state effective Hamiltonian $\boldsymbol{H}^{\text {eff }}=\boldsymbol{H}^{(0)}+\tilde{\boldsymbol{\Delta}}^{\mathrm{sBk}}$. Similar to the statespecific case, technically, one is able to store the vectors $\delta^{\mathrm{Bk}}$ and $\boldsymbol{c}^{(0)}$, but $\tilde{\Delta}^{\mathrm{sBk}}$ (or $\Delta^{\mathrm{sBk}}$ ) is potentially too large to be stored in memory. Luckily, compared to a standard CI calculation, the Davidson diagonalization procedure only requires, at each iteration, the extra knowledge of

$$
\tilde{\boldsymbol{\Delta}}^{\mathrm{sBk}} \boldsymbol{U}=\left(\boldsymbol{c}^{(0) \dagger} \boldsymbol{\delta}^{\mathrm{sBk}} \boldsymbol{U}+\boldsymbol{\delta}^{\mathrm{sBk} \dagger} \boldsymbol{c}^{(0)} \boldsymbol{U}\right) / 2,
$$

where $\boldsymbol{U}$ is a $N_{\text {det }} \times N_{\text {dav }}$ matrix gathering the $N_{\text {dav }}$ vectors considered in the Davidson diagonalization algorithm at a given iteration (with $N_{\text {st }} \leq N_{\text {dav }} \ll N_{\text {det }}$ ). Thanks to Eq. (14), this term can be efficiently evaluated in a $\mathcal{O}\left(N_{\text {det }}\right)$ computational cost and storage via two successive matrix multiplications, for instance,

$$
\boldsymbol{c}^{(0) \dagger} \boldsymbol{\delta}^{\mathrm{sBk}} \boldsymbol{U}=\left[\boldsymbol{c}^{(0)} \times\left({ }^{\dagger} \boldsymbol{\delta}^{\mathrm{sBk}} \times \boldsymbol{U}\right)\right] .
$$

A pseudo-code of our iterative multi-state dressing algorithm is presented in the supplementary material. For $N_{\text {st }}=1$, the present multi-state algorithm reduces to the state-specific version.

\section{HYBRID STOCHASTIC/DETERMINISTIC DRESSINGS}

In Ref. 17, we proposed to express

$$
E^{(2)}=\sum_{I=1}^{N_{\text {det }}} E_{[I]}^{(2)}
$$

as a sum of $N_{\text {det }}$ contributions $E_{[I]}^{(2)}$, each of them associated with a determinant of the model space, and to compute it efficiently via a Monte Carlo (MC) algorithm. Thanks to the relatively small size of the MC space $\left(N_{\mathrm{det}}\right)$, one is able to store each single contribution. Hence, during the MC simulation, if the contribution of a determinant is required and has never been computed previously, it is computed and stored. Otherwise, the value is retrieved from memory. This technique, known as memoization, drastically accelerates the MC calculation as each contribution needs to be computed only once. Moreover, we decompose the energy into a deterministic part and a stochastic part, making the deterministic part grow along the calculation until one reaches the desired accuracy. If desired, the calculation can be carried on until the stochastic part entirely vanishes. In that case, the exact result is obtained with no error bar and no noticeable computational overhead compared to the fully deterministic calculation. To summarize, this algorithm allows us to compute a truncated sum with no bias, but with a statistical error bar instead.

This algorithm is very general and is not limited to the calculation of $E^{(2)}$. Similar to Eq. (17), we express the dressing matrix (14) as the sum of dressing matrices

$$
\Delta^{\mathrm{sBk}}=\sum_{I=1}^{N_{\mathrm{det}}} \Delta_{[I]}^{\mathrm{sBk}} .
$$

Because the matrices $\Delta_{[I]}^{\mathrm{sBk}}$ are too large to fit in memory, we sample the vectors $\delta_{[I]}^{\mathrm{sBk}}$ [see Eq. (15b)], which are required for the Davidson diagonalization. During the sampling, one can monitor the "dressed" energy as

$$
E_{k}=\left\langle\Psi_{k}^{(0)}\left|\boldsymbol{H}_{k}^{\mathrm{eff}}\right| \Psi_{k}^{(0)}\right\rangle=E_{k}^{(0)}+{ }^{\dagger} \boldsymbol{c}^{(0)}\left\langle\boldsymbol{\delta}^{\mathrm{sBk}}\right\rangle,
$$

as well as its accuracy by computing the corresponding statistical error. In Sec. IV, all sBk calculations have been carried on until the statistical error is below $10^{-5}$ a.u. Let us emphasize once again that the primary purpose of the present MC algorithm is to accelerate the computation of the dressing matrix. The same results would have been obtained via its deterministic version.

\section{ILLUSTRATIVE CALCULATIONS}

Unless otherwise stated, all the calculations presented here have been performed with the electronic structure software QUANTUM PACKAGE, ${ }^{47}$ developed in our group and freely available. The sCI wave functions are generated with the CIPSI algorithm, as described in Refs. 1 and 3 in the frozencore approximation. The extrapolated FCI results, labeled as exFCI, have been obtained via the method recently proposed by Holmes, Umrigar, and Sharma ${ }^{9}$ in the context of the heatbath method. $^{7-9}$ This method has been shown to be robust even for challenging chemical situations, ${ }^{10-13}$ and we refer the interested readers to Ref. 11 for additional details.

\section{A. State-specific example}

To illustrate the improvement brought by the shifted-Bk approach in its state-specific version (see Sec. II A), we have computed the total electronic energy of the ${ }^{2} \Pi_{g}$ ground state of $\mathrm{CuCl}_{2}$ with the $6-31 \mathrm{G}$ basis set. The geometry has been taken from Ref. 2 where additional information can be found on this system. For this particular example, we have chosen a small basis set in order to be able to easily reach the FCI limit. A larger basis set will be considered in the next (multi-state) example (see Sec. IV B). The molecular orbitals have been obtained at the restricted open-shell Hartree-Fock (ROHF) level, and the 15 lowest doubly occupied orbitals have been frozen. This corresponds to a sCI calculation of 33 electrons in 38 orbitals. sCI-PT2 stands for a sCI calculation where we have added to the (zeroth-order) variational energy $E^{(0)}$ defined in Eq. (2) the value of the second-order correction $E^{(2)}$ given 


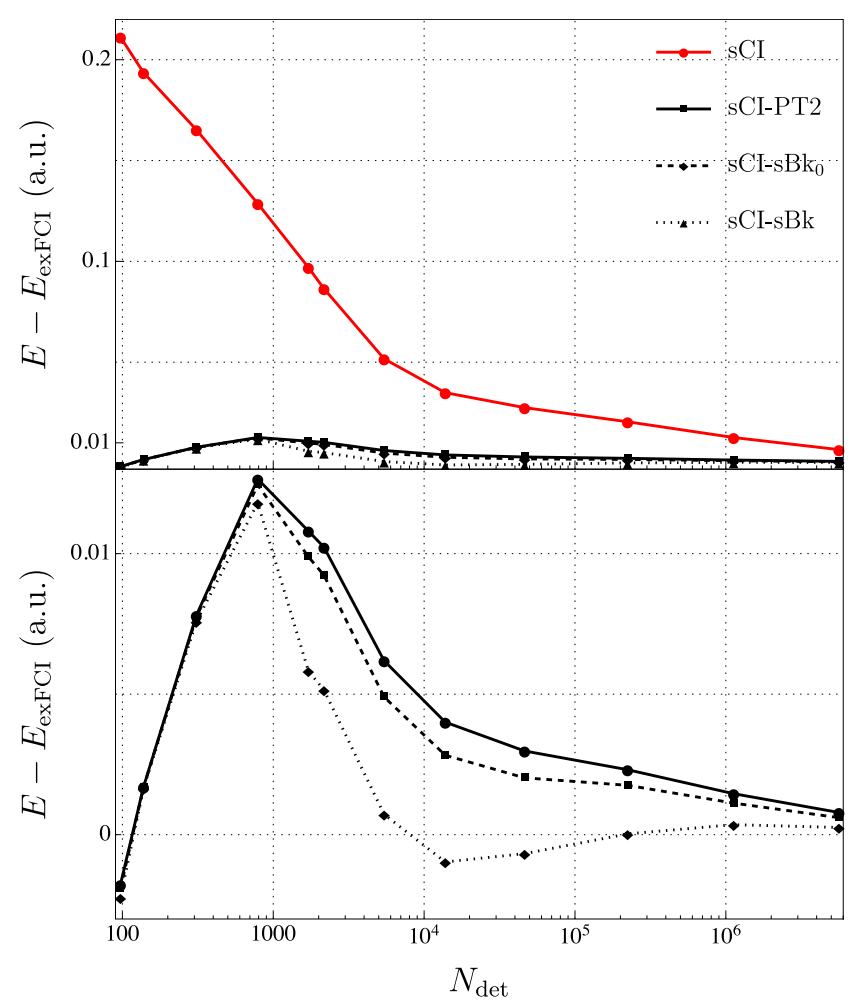

FIG. 1. Deviation from the extrapolated FCI energy $E_{\text {exFCI }}$ of the total energy $E$ of $\mathrm{CuCl}_{2}$ (in hartree) as a function of the number of determinants $N_{\text {det }}$ in the sCI wave function for various methods.

by Eq. (4). The one-shot non-iterative shifted-Bk procedure will be labeled as $\mathrm{sCI}-\mathrm{sBk}_{0}$, while its self-consistent version is simply labeled as sCI-sBk.

Figure 1 shows the convergence of the total energy of $\mathrm{CuCl}_{2}$ as a function of the number of determinants $N_{\text {det }}$ in the sCI wave function for the variational sCI results, as well as sCI-PT2, sCI-sBk , $_{0}$ and sCI-sBk. The corresponding numerical values are reported in Table I. As expected, the sCI-PT2, sCI-sBk 0 , and sCI-sBk energies are not variational as perturbative energies and energies obtained by projection are not guaranteed to be an upper bound of the FCI energy. Nonetheless, all of these corrections drastically improve the rate of convergence compared to the variational sCI results (note the logarithmic scale in Fig. 1). As shown in the bottom graph of Fig. 1, for small values of $N_{\text {det }}$, the three methods yield very similar total energies. However, for $N_{\text {det }} \gtrsim 10^{3}$, results start to deviate due to the inclusion of an important configuration corresponding to a ligand-to-metal charge transfer (LMCT) state. ${ }^{48}$ This LMCT configuration induces a strong revision of the model space wave function $\Psi^{(0)}$. Because the LMCT configuration corresponds to a singly excited determinant with respect to the ROHF determinant, it is not included in the CIPSI expansion for small $N_{\text {det }}$ values as it does not directly interact with the ROHF reference $\left(a^{2} b \rightarrow a b^{2}\right.$ excitation for which Brillouin's theorem does apply $\left.{ }^{49}\right)$. Therefore, the double excitations which are strongly coupled with the ROHF configuration are first selected by the CIPSI algorithm. Then, the LMCT configuration is included via its connection with the doubles. In particular, the double excitations corresponding to a single excitation on top of the LMCT configuration have been found to strongly interact with it. The key observation here is that the sCI-sBk energy converges much faster to the FCI limit than the sCI-PT2 energy. Moreover, the significant difference between sCI-sBk and sCI-sBk highlights the importance of the revision of the internal wave function brought by the self-consistent nature of the shifted-Bk method.

Table I also reports the overlap of the sCI and sCI-sBk wave functions with respect to the largest sCI wave function obtained for $N_{\text {det }}=26493179$. These results also highlight the faster convergence of sCI-sBk and illustrate that the shifted-Bk

TABLE I. Deviation (in millihartree) from the extrapolated FCI energy $\left(E_{\text {exFCI }}=-2558.006880\right.$ a.u.) for various methods as a function of the number of determinants $N_{\text {det }}$ in the CIPSI expansion for the $\mathrm{CuCl}_{2}$ molecule and the 6-31G basis set. The second-order correction $E^{(2)}$ is also reported. The error bar corresponding to one standard deviation is reported in parentheses. The exFCI energy has been obtained via a linear extrapolation using the energies of the two largest wave functions (see the supplementary material). The two rightmost columns report the overlap with respect to the largest sCI wave function.

\begin{tabular}{|c|c|c|c|c|c|c|}
\hline \multirow[b]{2}{*}{$N_{\text {det }}$} & \multirow[b]{2}{*}{$E^{(2)}$} & \multicolumn{3}{|c|}{$\Delta E$} & \multicolumn{2}{|c|}{ Overlap } \\
\hline & & sCI-PT2 & sCI-sBk ${ }_{0}$ & sCI-sBk & $\mathrm{sCI}$ & sCI-sBk \\
\hline 97 & $-213.039(0)$ & $-1.778(0)$ & $-1.93(0)$ & $-2.25(0)$ & 0.9275 & 0.9275 \\
\hline 138 & $-191.914(0)$ & $+1.698(0)$ & $+1.68(0)$ & $+1.65(0)$ & 0.9295 & 0.9295 \\
\hline 309 & $-157.491(0)$ & $+7.799(0)$ & $+7.74(0)$ & $+7.59(0)$ & 0.9345 & 0.9345 \\
\hline 789 & $-116.025(0)$ & $+12.654(0)$ & $+12.45(0)$ & $+11.81(0)$ & 0.9438 & 0.9447 \\
\hline 1708 & $-86.208(2)$ & $+10.807(2)$ & $+9.89(0)$ & $+5.83(0)$ & 0.9579 & 0.9671 \\
\hline 2167 & $-76.249(8)$ & $+10.232(8)$ & $+9.23(1)$ & $+5.15(1)$ & 0.9610 & 0.9700 \\
\hline 5428 & $-45.49(3)$ & $+6.19(3)$ & $+4.90(3)$ & $+0.72(3)$ & 0.9777 & 0.9854 \\
\hline 13803 & $-30.87(9)$ & $+4.00(9)$ & $+2.83(9)$ & $-0.97(9)$ & 0.9853 & 0.9912 \\
\hline 46327 & $-24.48(9)$ & $+2.98(9)$ & $+2.02(9)$ & $-0.68(9)$ & 0.9913 & 0.9952 \\
\hline 223089 & $-18.13(9)$ & $+2.31(9)$ & $+1.76(9)$ & $+0.03(9)$ & 0.9956 & 0.9975 \\
\hline 1125547 & $-11.18(9)$ & $+1.46(9)$ & $+1.12(9)$ & $+0.36(9)$ & 0.9984 & 0.9990 \\
\hline 5615264 & $-5.84(2)$ & $+0.79(2)$ & $+0.61(2)$ & $+0.26(2)$ & 0.9996 & 0.9997 \\
\hline 26493179 & $-3.34(2)$ & $+0.45(2)$ & $\ldots$ & $\ldots$ & 1.0000 & $\ldots$ \\
\hline
\end{tabular}


method could potentially provide better quality trial wave functions for quantum Monte Carlo. 1,2,5,6,11,13

Although $\Psi_{k}^{(0)}$ may be an eigenfunction of $\widehat{S}^{2}$, the way $\Psi_{k}^{(1)}$ is built does not enforce this property. The expectation value of $\widehat{S}^{2}$ can be monitored by

$$
\left\langle\Psi_{k}^{(0)}\left|\widehat{S}^{2}\right| \Psi_{k}^{(1)}\right\rangle={ }^{\dagger} \boldsymbol{c}_{k}^{(0) \dagger}\left(\boldsymbol{s}^{2}\right) \boldsymbol{c}_{k}^{(1)} .
$$

As expected, the deviation from the eigenvalue is always small, with a maximum deviation of the order of $10^{-4}$ a.u. in the case of $\mathrm{CuCl}_{2}$.

\section{B. Multi-state example}

We have chosen to illustrate the multi-state shifted$\mathrm{Bk}$ algorithm presented in Sec. II B by computing the first singlet transition energy of two cyanine dyes: $\mathrm{CN} 3$ $\left(\mathrm{H}_{2} \mathrm{~N}-\mathrm{CH}=\mathrm{NH}_{2}^{+}\right)$and $\mathrm{CN} 5\left(\mathrm{H}_{2} \mathrm{~N}-\mathrm{CH}=\mathrm{CH}-\mathrm{CH}=\mathrm{NH}_{2}^{+}\right)$. These types of dyes are known to be particularly challenging for electronic structure methods and especially timedependent density-functional theory. ${ }^{50-53}$ The geometry of CN5 has been extracted from Ref. 51 and we have optimized CN3 at the same level of theory (PBE0/cc-pVQZ). Here, we use Dunning's aug-cc-pVDZ basis set which has been shown to be flexible enough to quantitatively model such transitions thanks to the weak basis dependency of this valence $\pi \rightarrow \pi^{\star}$ transition. ${ }^{12,50}$ In order to treat the two singlet electronic states on equal footing, a common set of determinants is used for both states. In addition, state-averaged complete active space self-consistent field $\operatorname{CASSCF}(2,2)]$ molecular orbitals, obtained with the GAMESS package, ${ }^{54}$ are employed.

The difficulty of accurately modeling this vertical transition lies in the strong coupling between the $\sigma$ and $\pi$ spaces. To assess this peculiar effect, we have performed several calculations and our results are gathered in Table II. (The corresponding total energies can be found in the supplementary material.) For comparison purposes, Table II also reports reference calculations extracted from Ref. 50. First, we have performed CAS-CI calculations taking into account only the set of molecular orbitals with $\pi$ symmetry. We refer to these calculations as $\operatorname{CAS}(\pi)$. For $\mathrm{CN} 3$ and $\mathrm{CN} 5$, there are, respectively, 4 and 6 electrons as well as 32 and 50 orbitals in the $\operatorname{CAS}(\pi)$ space. This results in multideterminant wave functions containing 11296 and 670630 determinants, respectively. To quantify the strong coupling between the $\sigma$ and $\pi$ space, we have also computed full-valence exFCI energies [denoted as $\operatorname{exFCI}(\sigma+\pi)] .{ }^{11,12}$ These values fit nicely with the exCC3( $\sigma$ $+\pi$ ) benchmark values reported by Send et al., ${ }^{50}$ in agreement with our previous study which shows that, at least for compact compounds, CC3 and exFCI yield similar excitation energies. $^{12}$

The difference between $\operatorname{CAS}(\pi)$ and $\operatorname{exFCI}(\sigma+\pi)$ is of the order of half an $\mathrm{eV}$ (slightly less for CN5), showing that the relaxation of the $\sigma$ orbitals plays a central role here, this effect becoming less pronounced when the number of carbon atoms increases. Note that our $\operatorname{CAS}(\pi)$ excitation energies are extremely close to the CASSCF results reported in Table II. The diffusion Monte Carlo (DMC) estimates of Send et al. ${ }^{50}$ are probably off by $0.2 \mathrm{eV}$ due to the lack of direct $\sigma-\pi$
TABLE II. Vertical excitation energy (in eV) of cyanines for various methods. The error bar corresponding to one standard deviation is reported in parentheses.

\begin{tabular}{lccc}
\hline \hline Method & CN3 & CN5 & References \\
\hline $\operatorname{CAS}(\pi)^{\mathrm{a}}$ & 7.62 & 5.27 & This work \\
$\operatorname{CAS}(\pi)+\mathrm{PT} 2$ & 7.43 & 5.02 & This work \\
$\operatorname{CAS}(\pi)+\mathrm{sBk}_{0}$ & 7.40 & 4.98 & This work \\
$\operatorname{CAS}(\pi)+\mathrm{sBk}$ & 7.17 & 4.77 & This work \\
$\operatorname{exFCI}(\sigma+\pi)^{\mathrm{b}}$ & 7.17 & 4.89 & This work \\
\hline $\operatorname{CASSCF}(\pi)^{\mathrm{c}}$ & 7.59 & 5.25 & 50 \\
$\operatorname{CASPT} 2(\pi)^{\mathrm{d}}$ & 7.26 & 4.74 & 50 \\
$\operatorname{CC} 3(\sigma+\pi)^{\mathrm{e}}$ & 7.27 & 4.89 & 50 \\
\hline $\operatorname{DMC}$ & $7.38(2)$ & $5.03(2)$ & 50 \\
$\operatorname{exCC} 3(\sigma+\pi)^{\mathrm{g}}$ & 7.16 & 4.84 & 50 \\
\hline \hline
\end{tabular}

${ }^{\mathrm{a} C A S-C I / a u g-c c-p V D Z}$ calculations: CAS $(4,32)$ and CAS $(6,50)$ for CN3 and CN5, respectively.

${ }^{\mathrm{b}}$ Extrapolated CIPSI/aug-cc-pVDZ calculations (see the supplementary material).

${ }^{\mathrm{c} C A S S C F / A N O-L-V D Z P}$ calculations with optimal active spaces: CAS $(4,6)$ and $\mathrm{CAS}(6,10)$ for $\mathrm{CN} 3$ and $\mathrm{CN} 5$, respectively.

${ }^{\mathrm{d}}$ CASPT2/ANO-L-VDZP calculations with the standard IPEA Hamiltonian and optimal active spaces: $\mathrm{CAS}(4,6)$ and $\mathrm{CAS}(6,10)$ for $\mathrm{CN} 3$ and $\mathrm{CN} 5$, respectively.

${ }^{\mathrm{e}} \mathrm{CC} 3 / \mathrm{ANO}-\mathrm{L}-\mathrm{VDZP}$ excitation energies.

${ }^{\mathrm{f}}$ Diffusion Monte Carlo results based on optimal active space CASSCF trial wave functions obtained using the $\mathrm{T}^{\prime}+$ basis set and a Jastrow factor including electron-nuclear and electron-electron terms.

${ }^{\mathrm{g}}$ Extrapolated $\mathrm{CC} 3$ excitation energies obtained by adding the difference between the CC3/ANO-L-VDZP and CC2/ANO-L-VDZP values to the CC2/ANO-L-VTZP results.

coupling in the active space, which is only partially recovered by the Jastrow factor and the orbital optimization.

In $\operatorname{CAS}(\pi)+\mathrm{PT} 2$, the second-order correction $E^{(2)}$, computed by taking into account all the determinants from the FCI space connected to the $\operatorname{CAS}(\pi)$ reference space, is added to the $\operatorname{CAS}(\pi)$ result. This correction goes in the right direction and recovers 0.19 and $0.25 \mathrm{eV}$ for $\mathrm{CN} 3$ and $\mathrm{CN} 5$, respectively, bringing the excitation energies within 0.25 and $0.13 \mathrm{eV}$ to the $\operatorname{exFCI}(\sigma+\pi)$ values.

Similarly, $\mathrm{CAS}(\pi)+\mathrm{sBk}_{0}$ and $\mathrm{CAS}(\pi)+\mathrm{sBk}$ correspond to $\mathrm{sBk}$ and $\mathrm{sBk}_{0}$ calculations where the $\operatorname{CAS}(\pi)$ model space is renormalized by the effect of the perturbers. Like in the case of $\mathrm{CuCl}_{2}, \mathrm{CAS}(\pi)+\mathrm{sBk}_{0}$ recovers slightly more than $\operatorname{CAS}(\pi)+\mathrm{PT} 2$, while $\mathrm{CAS}(\pi)+\mathrm{sBk}$ is spot on for $\mathrm{CN} 3$ and overshoots slightly the $\operatorname{exFCI}(\sigma+\pi)$ values for CN5 with an error of $0.12 \mathrm{eV}$. These results show that the shifted-Bk method associated with a CIPSI-like sCI algorithm is able to recover a large fraction of the missing correlation energy, even with relatively small model spaces.

\section{SUPPLEMENTARY MATERIAL}

See supplementary material for the pseudo-code of the multi-state algorithm, total energies associated with Table II, and exFCI extrapolations.

\section{ACKNOWLEDGMENTS}

The authors would like to thank Jean-Paul Malrieu for stimulating discussions and the anonymous referees for valuable comments and suggestions. This work was performed using HPC resources from CALMIP (Toulouse) under 
allocations Nos. 2018-0510 and 2018-18005 and from GENCI-TGCC (Grant No. 2018-A0040801738).

${ }^{1}$ E. Giner, A. Scemama, and M. Caffarel, Can. J. Chem. 91, 879 (2013).

${ }^{2}$ M. Caffarel, E. Giner, A. Scemama, and A. Ramírez-Solís, J. Chem. Theory Comput. 10, 5286 (2014).

${ }^{3}$ E. Giner, A. Scemama, and M. Caffarel, J. Chem. Phys. 142, 044115 (2015).

${ }^{4}$ M. Dash, S. Moroni, A. Scemama, and C. Filippi, "Perturbatively Selected Configuration-Interaction Wave Functions for Efficient Geometry Optimization in Quantum Monte Carlo," J. Chem. Theory Comput. (published online).

${ }^{5}$ M. Caffarel, T. Applencourt, E. Giner, and A. Scemama, J. Chem. Phys. 144, 151103 (2016).

${ }^{6}$ M. Caffarel, T. Applencourt, E. Giner, and A. Scemama, "Using CIPSI nodes in diffusion Monte Carlo," in Recent Progress in Quantum Monte Carlo (ACS Publications, 2016), Chap. 2, pp. 15-46.

${ }^{7}$ A. A. Holmes, N. M. Tubman, and C. J. Umrigar, J. Chem. Theory Comput. 12, 3674 (2016).

${ }^{8}$ S. Sharma, A. A. Holmes, G. Jeanmairet, A. Alavi, and C. J. Umrigar, J. Chem. Theory Comput. 13, 1595 (2017).

${ }^{9}$ A. A. Holmes, C. J. Umrigar, and S. Sharma, J. Chem. Phys. 147, 164111 (2017).

${ }^{10}$ A. D. Chien, A. A. Holmes, M. Otten, C. J. Umrigar, S. Sharma, and P. M. Zimmerman, J. Phys. Chem. A 122, 2714 (2018).

${ }^{11}$ A. Scemama, Y. Garniron, M. Caffarel, and P. F. Loos, J. Chem. Theory Comput. 14, 1395 (2018).

${ }^{12}$ P. F. Loos, A. Scemama, A. Blondel, Y. Garniron, M. Caffarel, and D. Jacquemin, "A Mountaineering Strategy to Excited States: Highly Accurate Reference Energies and Benchmarks," J. Chem. Theory Comput. (published online).

${ }^{13}$ A. Scemama, A. Benali, D. Jacquemin, M. Caffarel, and P. F. Loos, J. Chem. Phys. 149, 034108 (2018).

${ }^{14}$ C. F. Bender and E. R. Davidson, Phys. Rev. 183, 23 (1969).

15 J. L. Whitten and M. Hackmeyer, J. Chem. Phys. 51, 5584 (1969).

${ }^{16}$ B. Huron, J. P. Malrieu, and P. Rancurel, J. Chem. Phys. 58, 5745 (1973).

${ }^{17}$ Y. Garniron, A. Scemama, P.-F. Loos, and M. Caffarel, J. Chem. Phys. 147, 034101 (2017).

${ }^{18}$ N. S. Blunt, J. Chem. Phys. 148, 221101 (2018).

${ }^{19}$ G. H. Booth, A. J. W. Thom, and A. Alavi, J. Chem. Phys. 131, 054106 (2009).

${ }^{20}$ F. A. Evangelista, J. Chem. Phys. 140, 124114 (2014).

${ }^{21}$ J. B. Schriber and F. A. Evangelista, J. Chem. Phys. 144, 161106 (2016).

${ }^{22}$ N. M. Tubman, J. Lee, T. Y. Takeshita, M. Head-Gordon, and K. B. Whaley, J. Chem. Phys. 145, 044112 (2016).
${ }^{23}$ W. Liu and M. R. Hoffmann, J. Chem. Theory Comput. 12, 1169 (2016).

${ }^{24}$ R. Cimiraglia, Int. J. Quantum Chem. 60, 167 (1996).

${ }^{25}$ J. P. Malrieu, P. Durand, and J. P. Daudey, J. Phys. A: Math. Gen. 18, 809 (1985).

${ }^{26}$ E. Giner, C. Angeli, Y. Garniron, A. Scemama, and J.-P. Malrieu, J. Chem. Phys. 146, 224108 (2017).

${ }^{27}$ S. Pathak, L. Lang, and F. Neese, J. Chem. Phys. 147, 234109 (2017).

${ }^{28}$ Z. Gershgorn and I. Shavitt, Int. J. Quantum Chem. 2, 751 (1968).

${ }^{29}$ L. E. Nitzsche and E. R. Davidson, J. Chem. Phys. 68, 3103 (1978).

${ }^{30}$ L. E. Nitzsche and E. R. Davidson, J. Am. Chem. Soc. 100, 7201 (1978).

${ }^{31}$ E. R. Davidson, L. E. McMurchie, and S. J. Day, J. Chem. Phys. 74, 5491 (1981).

${ }^{32}$ D. C. Rawlings and E. R. Davidson, Chem. Phys. Lett. 98, 424 (1983).

${ }^{33}$ D. C. Rawlings, E. R. Davidson, and M. Gouterman, Int. J. Quantum Chem. 26, 237 (1984).

${ }^{34}$ P. Kozlowski and E. Davidson, Chem. Phys. Lett. 226, 440 (1994).

${ }^{35}$ P. M. Kozlowski and E. R. Davidson, J. Chem. Phys. 100, 3672 (1994).

${ }^{36}$ P. M. Kozlowski and E. R. Davidson, Chem. Phys. Lett. 222, 615 (1994).

${ }^{37}$ P. M. Kozlowski, M. Dupuis, and E. R. Davidson, J. Am. Chem. Soc. 117, 774 (1995).

${ }^{38}$ V. N. Staroverov and E. R. Davidson, Chem. Phys. Lett. 296, 435 (1998).

${ }^{39}$ H. Nakano, J. Chem. Phys. 99, 7983 (1993).

${ }^{40}$ H. Nakano, J. Nakatani, and K. Hirao, J. Chem. Phys. 114, 1133 (2001).

${ }^{41}$ B. Kirtman, J. Chem. Phys. 75, 798 (1981).

${ }^{42}$ G. Li Manni, D. Ma, F. Aquilante, J. Olsen, and L. Gagliardi, J. Chem. Theory Comput. 9, 3375 (2013).

${ }^{43}$ P.-O. Löwdin, J. Chem. Phys. 19, 1396 (1951).

${ }^{44}$ P. Claverie, S. Diner, and J. P. Malrieu, Int. J. Quantum Chem. 1, 751 (1967).

${ }^{45}$ R. J. Buenker and S. D. Peyerimhoff, Theor. Chim. Acta 39, 217 (1975).

${ }^{46}$ E. R. Davidson, J. Comput. Phys. 17, 87 (1975).

${ }^{47}$ A. Scemama, T. Applencourt, Y. Garniron, E. Giner, G. David, and M. Caffarel, Quantum package v1.0, https://github.com/LCPQ/quantum_package, 2016.

${ }^{48}$ E. Giner and C. Angeli, J. Chem. Phys. 143, 124305 (2015).

${ }^{49}$ D. Feller and E. R. Davidson, J. Chem. Phys. 80, 1006 (1984).

${ }^{50}$ R. Send, O. Valsson, and C. Filippi, J. Chem. Theory Comput. 7, 444 (2011).

${ }^{51}$ D. Jacquemin, Y. Zhao, R. Valero, C. Adamo, I. Ciofini, and D. G. Truhlar, J. Chem. Theory Comput. 8, 1255 (2012).

${ }^{52}$ P. Boulanger, D. Jacquemin, I. Duchemin, and X. Blase, J. Chem. Theory Comput. 10, 1212 (2014).

${ }^{53}$ B. Le Guennic and D. Jacquemin, Acc. Chem. Res. 48, 530 (2015).

${ }^{54}$ M. W. Schmidt, K. K. Baldridge, J. A. Boatz, S. T. Elbert, M. S. Gordon, J. H. Jensen, S. Koseki, N. Matsunaga, K. A. Nguyen, S. Su et al., J. Comput. Chem. 14, 1347 (1993). 